\title{
AOR
}

Selected Papers of \#AolR2021:

The 22nd Annual Conference of the Association of Internet Researchers Virtual Event / 13-16 Oct 2021

\section{LIKES, SARCASM AND POLITICS: YOUTH RESPONSES TO A PLATFORM-INITIATED MEDIA LITERACY CAMPAIGN ON SOCIAL MEDIA}

\author{
loana Literat \\ Teachers College, Columbia University \\ Abubakr Abdelbagi \\ Teachers College, Columbia University \\ Nicola YL Law \\ Teachers College, Columbia University \\ Marcus Y-Y Cheung \\ Teachers College, Columbia University \\ Rongwei Tang \\ Teachers College, Columbia University

\section{Introduction}

Amidst an alarming rise in online misinformation, there has been a growing interest in media literacy education as a way to combat the rapid spread of misinformation (Bulger \& Davison, 2018). However, the focus of research and practice has been on the integration of media literacy instruction into young people's various educational experiences, rather than implementing and evaluating such initiatives on social media (Bulger \& Davison, 2018), which is known to be their central news source (Common Sense Media, 2019). Because of the lack a nuanced understanding of these dynamics on the platforms most popular with youth today, our study aimed to analyze how users responded to a platform-initiated media literacy education campaign on TikTok-an increasingly popular yet understudied social media platform - with a focus on the lessons that we might draw from these responses in terms of targeted media literacy education initiatives for youth. 


\section{Methodology}

While there is broad societal consensus about the urgency and significance of media literacy education, especially for young people, we still lack a grounded, bottom-up understanding of youth attitudes towards such initiatives. Moreover, trying to capture these attitudes through self-report methods like surveys or interviews runs the risk of producing biased results, due to social desirability effects (especially strong regarding media literacy and misinformation; see Tully et al., 2020), as well as the normative pressures that shape the way youth might respond to adult questions about their educational experiences. Here, using qualitative content analysis on a large corpus of youth reactions to a media literacy campaign on social media facilitates a naturalistic study of young people's attitudes towards such initiatives in situ, "in the wild."

A thematic analysis, particularly suitable for understanding experiences, views and perceptions (Braun \& Clarke, 2006), was conducted on 11,449 public comments posted in response to five videos created by TikTok US in partnership with external experts and TikTok microcelebrities. Videos were part of a strategic media literacy campaign aimed at youth and designed to highlight skills like fact-checking, assessing the credibility of information and visuals, understanding bias, and distinguishing facts from opinions. They were extremely popular on the platform, accumulating a total of $53.8 \mathrm{~m}$ views, $1.63 \mathrm{~m}$ likes, and $11 \mathrm{k}$ shares. Based on the age demographics of TikTok and the content of the comments themselves (e.g. references to school, use of youth lingo, references to youth culture), we can infer that the vast majority of commenters are youth.

\section{Findings}

Reactions to the campaign were mixed, and highly political in nature. While young people appreciated the urgency of media literacy education and understood its relevance to their social media participation, negative comments-often sarcastic in tone-far outweighed the more positive takes. These negative reactions were grounded in: confusion about the aim of the videos; annoyance at videos being pushed on users' "for you page"; disliking the video content and aesthetics; and perceived hypocrisy due to TikTok's own actions and policies. Particularly, they called out TikTok's failure to control the spread of misinformation on its platform and brought up stories of reporting questionable content, with TikTok taking no action about it.

Whether positive, negative, or neutral, a large proportion of comments were political, and addressed topics like Black Lives Matter, partisanship and the presidential election. Political comments usually got more responses than non-political ones, and exchanges were longer and more engaged, though often impolite and uncivil. Commenters frequently expressed their political identities and stances; a prevalent pattern was mentioning the opposing party in comments and saying that they need to watch these videos, thus implying that the other party/ideology is lacking media literacy skills. Significantly, the rationale behind the videos was politicized too, framing media literacy education as an inherently political endeavor. 


\section{Implications and Recommendations}

We identify key takeaways and recommendations that can valuably inform future media literacy campaigns on social media. First, youth were keenly aware of TikTok's "rap sheet" in regard to curbing misinformation (or not), which shaped by both micro- and macro-level factors (e.g. personal experience with reporting/moderation on the platform, mass media reports, political rhetoric). Commenters openly rebuked TikTok for sharing educational materials addressing fake news while failing to curb the spread of misinformation, which resulted in a diminished level of trust. Providing explicit reasoning around content moderation practices promotes a higher level of user trust (Brunk, et al., 2019), which might help with the effectiveness of such educational initiatives. Platforms should aim for increased transparency in communicating how decisions are made regarding user reports of misinformation or content violations.

Much of the negative feedback addresses promotion and design/content as being at odds with the ethos and function of TikTok in youth lives. While TikTok's campaign attempted to meet these expectations, users did not connect with the language and style employed in the videos. This might be understood in relation to the shift in TikTok users' aesthetic appetite, which is moving away from "visual theatrics" and towards more relatable content that taps into the current trends and latest memes (Abidin, 2021). We also found that youth were extremely frustrated with how the videos were being pushed onto their feeds. A key way to preempt these pitfalls is to include youth in the design of such campaigns via participatory design, which has been shown to be an effective strategy for the design of news literacy initiatives for youth (Literat, Chang \& Hsu, 2020). For instance, initiating participatory activities that align with the ethos of TikTok (e.g. TikTok challenges) and/or engaging influencers in the promotion of content.

Finally, the highly political nature of responses is a powerful illustration of the politicized nature of media literacy education in the current sociocultural landscape. Future media literacy initiatives should be aware that even apolitical content might be politicized and framed in polarizing terms by users. These findings also reinforce the link between media literacy and civic education (Mihailidis, 2018), and, particularly in this political and technological context, each is a prerequisite for the other.

\section{References}

Abidin, C., 2021. Mapping internet celebrity on TikTok: Exploring attention economies and visibility labours. Cultural Science Journal, 12(1), 77-103.

https://doi.org/10.5334/csci.140

Braun, V. and Clarke, V. (2006) Using thematic analysis in psychology. Qualitative Research in Psychology, 3(2), 77-101.

Brunk, J., Mattern, J. and Riehle, D.M. (2019). Effect of transparency and trust on acceptance of automatic online comment moderation systems. In IEEE 21st Conference on Business Informatics (CBI) (Vol. 1, pp. 429-435). IEEE.

https://doi.org/10.1109/CBI.2019.00056. 
Bulger, M., \& Davison, P. (2018). The promises, challenges, and futures of media literacy. Journal of Media Literacy Education, 10(1), 1-21.

https://doi.org/10.23860/JMLE-2018-10-1-1

Common Sense Media (2019). Teen news engagement: Key findings and toplines. https://www.commonsensemedia.org/sites/default/files/uploads/pdfs/2019 cssm summarytoplines release.pdf

Literat, I., Chang, Y. K., \& Hsu, S. Y. (2020). Gamifying fake news: Engaging youth in the participatory design of news literacy games. Convergence, 26(3), 503-516. https://doi.org/10.1177\%2F1354856520925732

Mihailidis, P. (2018). Civic media literacies: re-imagining engagement for civic intentionality. Learning, Media and Technology, 43( 2), 152-164.

https://doi.org/10.1080/17439884.2018.1428623 